\title{
The impact of obesity on early postoperative atrial fibrillation burden
}

Corina Serban, DVM, ${ }^{\mathrm{a}}$ Johnmary T. Arinze, MBBS, ${ }^{\mathrm{a}}$ Roeliene Starreveld, MSc, ${ }^{\mathrm{a}}$ Eva A. H. Lanters, MD, ${ }^{\mathrm{a}}$ Ameeta Yaksh, MD, ${ }^{\mathrm{b}}$ Charles Kik, MD,${ }^{\mathrm{c}}$ Yalin Acardag, BSc, ${ }^{\mathrm{a}}$ Paul Knops, BSc, ${ }^{\mathrm{a}}$ Ad J. J. C. Bogers, MD, PhD, ${ }^{c}$ and Natasja M. S. de Groot, MD, $\mathrm{PhD}^{\mathrm{a}}$

\section{ABSTRACT}

Background: Obesity has been linked to the development of postoperative atrial fibrillation. This study is aimed at investigating the role of body mass index in the evolution of de novo, early postoperative atrial fibrillation by assessing differences between obese and nonobese patients undergoing cardiac surgery.

Methods: Patients with early de novo postoperative atrial fibrillation were included. Continuous cardiac rhythms were recorded during the first 5 postoperative days in obese $(\mathrm{N}=67,66 \pm 9$ years; $51[76 \%]$ male $)$ and nonobese $(\mathrm{N}=89$, $69 \pm 9 ; 75[84 \%$ ] male) patients without a history of atrial fibrillation undergoing cardiac surgery. Postoperative atrial fibrillation burden was defined as the ratio between total duration of all atrial fibrillation episodes and total recording time (atrial fibrillation burden, \%).

Results: A total of 1191 (median: 5/patient) postoperative atrial fibrillation episodes were identified in the obese group compared with 1218 (median: 4/patient) in the nonobese group. The median duration and number of prolonged ( $>60$ minutes) postoperative atrial fibrillation episodes were higher in obese patients (250 vs 145 minutes, $P=.003$, and median of 2 vs 1 episode, $P=.031$ ). Obesity was associated with a larger early postoperative atrial fibrillation burden (obese patients: median, 7\% interquartile range, 2.5-19.7 vs nonobese patients: median, $3.2 \%$; interquartile range, $0.5-8.8, P=.001)$ mainly on the third postoperative day $(P=.021)$.

Conclusions: Obesity predisposes to a larger number of prolonged atrial fibrillation episodes in the early postoperative period after cardiac surgery for coronary artery disease or valvular heart disease. The higher atrial fibrillation burden in the early postoperative period occurred particularly on the third day. Future studies will determine whether obesity prevention may play a key role in reducing the incidence of postoperative atrial fibrillation in patients undergoing cardiac surgery. (J Thorac Cardiovasc Surg 2020;159:930-8)

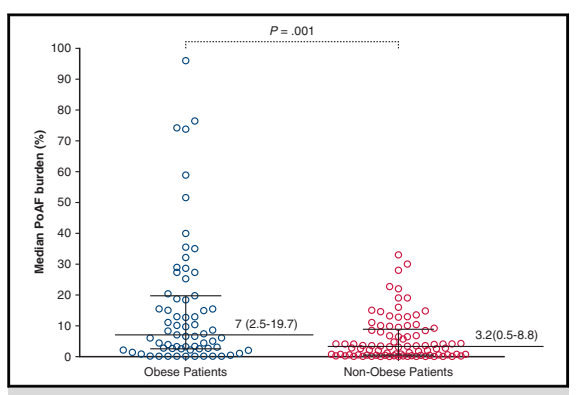

Median PoAF burden (\%) in obese and nonobese patients $(P=.001)$

\section{Central Message}

Obese patients have a higher incidence of particularly long-lasting episodes of early PoAF and a higher burden for early PoAF compared with nonobese patients.

\section{Perspective}

Obesity has been linked to development of PoAF. Our study shows obesity predisposes to a larger number of prolonged $\mathrm{AF}$ episodes and a higher AF burden in the early postoperative period after cardiac surgery. Because the effects of obesity on the AF substrate are reversible with weight control, preventive lifestyle before cardiac surgery may play an invaluable role.

See Commentaries on pages 939 and 941.
From the Departments of a Cardiology and ${ }^{\mathrm{c} C}$ ardiothoracic Surgery, Erasmus University Medical Center, Rotterdam, The Netherlands; and ${ }^{\mathrm{b}}$ Department of Cardiology, Radboud University, Nijmegen, The Netherlands.

N.M.S.dG. is supported by grants from the Erasmus Medical Center fellowship, Dutch Heart Foundation (2012T0046), LSH-Impulse Grant 40-43100-98-008, CVON AFFIP (Grant No. 914728), and VIDI Grant (No. 91717339).

Received for publication Aug 24, 2018; revisions received March 15, 2019; accepted for publication March 18, 2019; available ahead of print April 29, 2019.

Address for reprints: Natasja M.S. de Groot, MD, PhD, Department of Cardiology, Erasmus University Medical Center, Doctor Molewaterplein 40, 3015 GD Rotterdam, The Netherlands (E-mail: n.m.s.degroot@erasmusmc.nl).

$0022-5223 / \$ 36.00$

Copyright (c) 2019 by The American Association for Thoracic Surgery

https://doi.org/10.1016/j.jtcvs.2019.03.073
Obesity is a worldwide epidemic affecting people of all ages. There is strong evidence that obesity is a predictor for the development of atrial fibrillation (AF). ${ }^{1-4}$ The association between obesity and evolution of $\mathrm{AF}$ is

Scanning this QR code will take you to the article title page to access supplementary information.

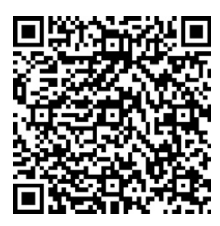



Abbreviations and Acronyms
$\mathrm{AF}=$ atrial fibrillation
$\mathrm{BMI}=$ body mass index
$\mathrm{CAD}=$ coronary artery disease
$\mathrm{DM}=$ diabetes mellitus
EAT $=$ epicardial adipose tissue
$\mathrm{IQR}=$ interquartile range
$\mathrm{PoAF}=$ postoperative atrial fibrillation

multifactorial and still incompletely elucidated. Prior studies have demonstrated that metabolic syndrome, ischemic heart disease, local and systemic inflammatory processes, epicardial fat distribution, cardiac remodeling, and genetic factors ${ }^{3,5}$ play an important role in $\mathrm{AF}$ development. In mice, obesity is associated with reversible ionic remodeling of the left atrial appendage, resulting in higher incidence of AF inducibility and burden. ${ }^{6,7}$

Obesity is becoming more prevalent in patients undergoing cardiac surgery, particularly in those referred for coronary artery bypass grafting, and is associated with an increase in short- and long-term postoperative morbidity and mortality. ${ }^{4,8-12}$ De novo postoperative atrial fibrillation (PoAF) is a common complication that occurs more frequently in obese patients. ${ }^{13-16}$ However, characteristics of de novo early PoAF episodes, including the number and duration of PoAF episodes, in obese patients have not been investigated.

We investigated the role of body mass index (BMI) in the characteristics of de novo PoAF by assessing differences between obese and nonobese patients undergoing cardiac surgery with regard to early PoAF episodes.

\section{MATERIALS AND METHODS}

This study was conducted as part of 2 prospective observational projects, including Quest for Arrhythmogenic Substrate of Atrial fibrRillation (QUASAR, MEC 2010-054) and Hsf1 Activators Lower cardiomyocyte damage Towards a novel approach to REVERSE atrial fibrillation (HALT \& REVERSE, MEC 2014-393). Both projects were approved by the local ethics committee of the Erasmus Medical Centre and adhere to the Declaration of Helsinki principles. Accordingly, written consent was obtained from participating patients before surgical intervention.

\section{Study Population}

The study population consisted of adult patients scheduled for elective cardiac surgery for coronary artery disease (CAD), either isolated or in combination with aortic (CAD + aortic valve disease) or mitral valve disease (CAD + mitral valve disease), isolated aortic valve disease, or mitral valve disease. Patients presenting with early PoAF were included. Exclusion criteria were history of AF, prior ablation of atrial tachyarrhythmias, severe renal failure, and patients with an atrial pacing device and requiring mechanical or inotropic support. Patients scheduled for elective cardiac surgery for congenital heart disease were also excluded. The sample was divided into 2 categories: (1) obese patients (BMI $\geq 30 \mathrm{~kg} / \mathrm{m}^{2}$ ) and (2) nonobese patients (BMI $<30 \mathrm{~kg} / \mathrm{m}^{2}$ ).

\section{Automatic Detection of Early Postoperative Atrial Fibrillation}

Cardiac rhythms of every patient were continuously recorded during the first 5 postoperative days using bedside monitors (Draeger Infinity, Draeger Inc, Telford, Pa). All postoperative continuous rhythm registrations (.CPZ) were converted into a MATLAB-compatible format (.ECG) before analysis (MathWorks, Natick, Mass). Algorithms were used to detect the start and ending of each AF episode from the moment of arrival on the surgical ward to the end of postoperative day 5. All PoAF episodes detected by the software were cross-checked in Synescope (Livanova, London, England) by 2 blinded operators to eliminate potential false-positive registrations induced by artefacts. Electrocardiograms and patients records were manually evaluated for the presence of AF during the first 5 days of postoperative hospitalization.

\section{Characterization of Postoperative Atrial Fibrillation}

PoAF was defined as a series of supraventricular beats with irregularly irregular R-R intervals, in the absence of P-waves, persisting for 30 seconds or more. For every patient, the number and duration of AF episodes in the continuous rhythm registrations were quantified manually and used to calculate the PoAF burden during the first 5 postsurgical days. The PoAF burden was defined as the ratio between the total duration of all AF episodes and the total recording time (AF burden $[\%]=$ total time spent in $\mathrm{AF}[$ minutes]/recording time [minutes] $* 100$ ).

\section{Antiarrhythmic Treatment for Early De Novo Postoperative Atrial Fibrillation}

On the first postoperative day, all patients received an oral beta-blocker, starting with a dose of $25 \mathrm{mg}$ twice daily. Patients with PoAF were cardioverted to sinus rhythm (SR) within 48 hours using intravenous bolus of amiodarone $300 \mathrm{mg}$. Amiodarone was discontinued when AF converted to SR. If conversion was not achieved within less than 48 hours from PoAF onset, electrical cardioversion was performed, and anticoagulant therapy was started combined with oral amiodarone $200 \mathrm{mg} 3$ times daily for another 4 days. Patients who were hemodynamically unstable at PoAF onset were electrically cardioverted to SR followed by 4 days of oral amiodarone $200 \mathrm{mg} 3$ times daily. If AF was accepted, the beta-blocker dosage was increased if the heart rate was greater than 110 beats/min.

\section{Statistical Analysis}

Statistical analysis was performed using IBM SPSS Statistics 24 software (SPSS, Armonk, NY). Data were tested using Shapiro-Wilk test of normality. Continuous, normally distributed data are expressed as mean \pm standard deviation, and skewed data as median and interquartile range (IQR) (P25/P75). Student $t$ test was used to compare normally distributed continuous variables, and skewed parameters were compared using the Mann-Whitney $U$ test. Comparisons between related skewed variables were performed using the Wilcoxon test. Variability between and within groups was assessed using analysis of variance. Categoric variables were compared using the chi-square test and are presented as percentages. Bonferroni correction was used to adjust $P$ value for multiple comparisons. Possible factors associated with early PoAF burden were introduced in a linear regression analysis. PoAF burden was transformed using the logarithm function. The variables manually introduced in the linear regression model included all the baseline clinical characteristics presented in Table 1, provided that the model maintained significance. Pearson correlation test was used to evaluate the linear relationship between continuous variables.

\section{RESULTS}

\section{Study Population}

Baseline characteristics of both the obese group $(\mathrm{N}=67$, $66 \pm 9$ years; $51[76 \%]$ male $)$ and nonobese group $(\mathrm{N}=89$, 
TABLE 1. Patient characteristics

\begin{tabular}{|c|c|c|c|}
\hline Risk factors & $\begin{array}{c}\text { Obese patients } \\
\text { BMI } \geq 30 \mathrm{~kg} / \mathrm{m}^{2} \\
(\mathrm{~N}=67)\end{array}$ & $\begin{array}{c}\text { Nonobese patients } \\
\text { BMI }<30 \mathrm{~kg} / \mathrm{m}^{2} \\
(\mathrm{~N}=\mathbf{8 9})\end{array}$ & $P$ value \\
\hline Age $(y)$ & $66.1 \pm 9.7$ & $69.1 \pm 9.2$ & .027 \\
\hline Male gender, N (\%) & $51(76)$ & $75(84)$ & .223 \\
\hline BMI & $32.4 \pm 2.3$ & $25.2 \pm 4.3$ & $<.001$ \\
\hline \multicolumn{4}{|l|}{ Risk factors, $\mathrm{N}(\%)$} \\
\hline Hypertension & $34(50)$ & $42(47)$ & .747 \\
\hline $\mathrm{DM}$ & $21(31.3)$ & $12(13.4)$ & .018 \\
\hline Dyslipidemia & $19(28.3)$ & $36(40.4)$ & .130 \\
\hline Left ventricular function & & & .361 \\
\hline Normal $(\mathrm{EF}>55 \%)$ & $42(62.6)$ & $62(63.2)$ & \\
\hline Mild impairment (EF 46\%-55\%) & $15(22.3)$ & $21(23.5)$ & \\
\hline Moderate impairment (EF 36\%-45\%) & $5(7.5)$ & $4(4.5)$ & \\
\hline Severe impairment $(E F<35 \%)$ & $5(7.5)$ & $2(2.24)$ & \\
\hline LA dilatation, $N(\%)$ (LA diameter $\geq 45 \mathrm{~mm}$ ) & $14(21)$ & $16(18)$ & .205 \\
\hline CHA2DS2-VASc score, $\mathrm{N}(\%)$ & & & .229 \\
\hline Low $($ CHA2DS2-VASc score $=0$ ) & $5(7.4)$ & $10(11.2)$ & \\
\hline Low-moderate $(\mathrm{CHA} 2 \mathrm{DS} 2-\mathrm{VASc}$ score $=1)$ & $13(19.4)$ & $27(30.3)$ & \\
\hline Moderate-high (CHA2DS2-VASc score $\geq 2$ ) & $49(73.1)$ & $52(58.4)$ & \\
\hline \multicolumn{4}{|l|}{ Preoperative medication, $\mathrm{N}(\%)$} \\
\hline \multicolumn{4}{|l|}{ Antiarrhythmic drugs } \\
\hline Class II & $46(68.6)$ & $59(66.2)$ & .863 \\
\hline Class IV & $2(3)$ & $4(4.4)$ & .467 \\
\hline ACE inhibitors/II & $42(62.6)$ & $49(55)$ & .412 \\
\hline Surgical procedure, $\mathrm{N}(\%)$ & & & .307 \\
\hline CABG & $42(62.6)$ & $50(56)$ & \\
\hline AVD & $11(16.4)$ & $10(11.2)$ & \\
\hline MVD & $4(6)$ & $5(5.6)$ & \\
\hline $\mathrm{CABG}+\mathrm{AVD}$ & $8(11.9)$ & $14(15.7)$ & \\
\hline $\mathrm{CABG}+\mathrm{MVD}$ & $2(3)$ & $10(11.2)$ & \\
\hline
\end{tabular}

Statistically significant values $(P<.05)$. BMI, Body mass index; $D M$, diabetes mellitus; $E F$, ejection fraction; $L A$, left atrial; $C H A 2 D S 2$-VASC, Congestive heart failure, Hypertension, Age, Diabetes, Stroke-Vascular disease; $A C E$, angiotensin-converting enzyme; $C A B G$, coronary artery bypass grafting; $A V D$, aortic valve disease; $M V D$, mitral valve disease.

$69 \pm 9 ; 75[84 \%]$ male) are presented in Table 1 . Clinical characteristics between the obese and nonobese groups only differed in age $(66 \pm 9.7$ years vs $69 \pm 9.2$ years; $P=.024)$, BMI $(32.4 \pm 2.3$ vs $25.2 \pm 4.3 ; P<.001)$, and incidence of diabetes mellitus (DM) $(\mathrm{N}=21[31.3 \%]$ vs $\mathrm{N}=12[13.4 \%] ; P=.01)$. Coronary artery bypass grafting was the main surgical procedure performed in both groups (42 [63\%] obese patients and $50[56 \%]$ nonobese patients).

\section{Duration of Postoperative Atrial Fibrillation Episodes}

In the postoperative rhythm registrations, 1191 PoAF episodes were identified in the obese group and 1218 in the nonobese group. Characteristics of PoAF episodes for obese and nonobese patients are depicted in Table 2. In the obese and nonobese groups, the median number of PoAF episodes per patient was 5 (IQR, 2-16) and 4 (IQR, 2-15), respectively.
Figure 1 shows that the median duration of PoAF episodes was higher in obese patients (median 294.5 minutes; IQR, 105.5-1036.5) compared with nonobese patients (median 182.5 minutes; IQR, 22.5-498.3, $P=.008$ ).

The median duration of PoAF episodes lasting longer than 60 minutes is depicted in Figure 2. There was a significant increase in the median duration of prolonged ( $>60$ minutes) PoAF episodes in obese patients ( $250 \mathrm{mi}-$ nutes; IQR, 135-421) compared with nonobese patients (145.4 minutes; IQR, 97.4-252.4) $(P=.003)$. For episodes lasting between 30 seconds and 1 hour, no significant change in median duration of PoAF episodes was found between obese and nonobese patients.

Figure 3 depicts the median number of PoAF episodes for the obese and nonobese patients separately. As demonstrated in Figure 3, obese patients had significantly more prolonged PoAF episodes (episodes lasting $>60$ minutes) compared with nonobese patients $(P=.031)$. Figure 3 
TABLE 2. Characteristics of postoperative atrial fibrillation episodes

\begin{tabular}{lccccc}
\hline & \multicolumn{2}{c}{ Obese patients } & \multicolumn{2}{c}{ Nonobese patients } \\
\cline { 2 - 3 } Time (min) & $\begin{array}{c}\text { No. of PoAF episode(s) } \\
\text { Total, median (IQR) }\end{array}$ & $\begin{array}{c}\text { Duration (min) of PoAF episode(s) } \\
\text { Total, median (IQR) }\end{array}$ & $\begin{array}{c}\text { No. of PoAF episode(s) } \\
\text { Total, median (IQR) }\end{array}$ & $\begin{array}{c}\text { Duration (min) of PoAF episode(s) } \\
\text { Total, median (IQR) }\end{array}$ \\
\hline $0-5$ & $746 ; 3.5(1-14)$ & $89.25 ; 1.5(1-2.5)$ & $834 ; 7(2-14)$ & $94 ; 1.5(1-2)$ \\
\hline $5.5-10$ & $128 ; 2(1-5)$ & $190.25 ; 7(6-7.25)$ & & $116 ; 2(1-5)$ & $238.5 ; 7(6-8.5)$ \\
\hline $10.5-15$ & $55 ; 1(1-4)$ & $265 ; 12.75(12-13.5)$ & & $62 ; 2(1-4)$ & $275.75 ; 12.75(11.43-13.5)$ \\
\hline $15.5-20$ & $36 ; 1(1-2.75)$ & $284.5 ; 17.6(17-18.5)$ & $20 ; 1(1-2)$ & $351 ; 19(17.75-19.75)$ \\
\hline $20.5-25$ & $20 ; 1(1-2.5)$ & $198.5 ; 22(21.25-22.75)$ & $19 ; 1(1-2)$ & $245 ; 21.5(20.5-23.75)$ \\
\hline $25.5-30$ & $12 ; 1$ & $299.75 ; 27(26-28)$ & $13 ; 1$ & $303 ; 27(26-28.5)$ \\
\hline $30.5-35$ & $20 ; 1(1-2)$ & $390.2 ; 33(31-33.5)$ & $8 ; 1$ & $229.5 ; 32.5(32-33.5)$ \\
\hline $35.5-40$ & $14 ; 1(1-2)$ & $407.25 ; 36.5(36.5-38.5)$ & & $10 ; 1$ & $378 ; 38.25(36.25-39.5)$ \\
\hline $40.5-45$ & $11 ; 1$ & $425.25 ; 42.3(41.87-43.25)$ & $9 ; 1(1-2)$ & $300.5 ; 42.75(42-44)$ \\
\hline $45.5-50$ & $6 ; 1(1-5)$ & $240.5 ; 49(46.25-49.5)$ & & $5 ; 1$ & $338.5 ; 49(47-50)$ \\
\hline $50.5-55$ & $9 ; 1$ & $422 ; 52.5(51.5-54)$ & $10 ; 1$ & $311.75 ; 51.37(50.5-53.5)$ \\
\hline $55.5-60$ & $7 ; 1(1-2)$ & $266 ; 56.5(56.1-56.8)$ & & $14 ; 1(1-1.5)$ & $518.75 ; 58(56.5-58.6)$ \\
\hline 60 & $120 ; 1(1-3)$ & $15678.7 ; 250.5(135-421.25)$ & $96 ; 1(1-2)$ & $10314.5 ; 145.37(97.43-252.43)$ \\
\hline
\end{tabular}

The number and duration of PoAF episodes are categorized according to their durations. PoAF, Postoperative atrial fibrillation; IQR, interquartile range.

shows no significant difference in the median number of PoAF episodes lasting less than 1 hour between obese and nonobese patients.

In obese patients, the majority of PoAF episodes (746 [62.6\%], median 3.5; IQR, 1-14) terminated within 5 minutes; 318 episodes (27\%, median 1; IQR, 1.0-3.7) lasted between 5 and 60 minutes, and 120 PoAF episodes (10\%, median 2; IQR, 1-4) had a duration longer than 1 hour.

In nonobese patients, most PoAF episodes (834, 68.4\%, median 7; IQR, 2-14) also terminated within 5 minutes, and
286 episodes (23\%, median 1; IQR 1.0-3.7) lasted between 5 minutes and 1 hour. However, only 96 PoAF episodes (8\%, median 1; IQR, 1-2) had a duration longer than 60 minutes.

\section{Burden of Postoperative Atrial Fibrillation Episodes}

The PoAF burden for each patient individually is plotted in Figure 4 and shows that the PoAF burden is larger in the obese patients (median 7\%; IQR, 2.5-19.7) compared with the nonobese patients (median 3.2\%; IQR, 0.5-8.8) $(P=.001)$.

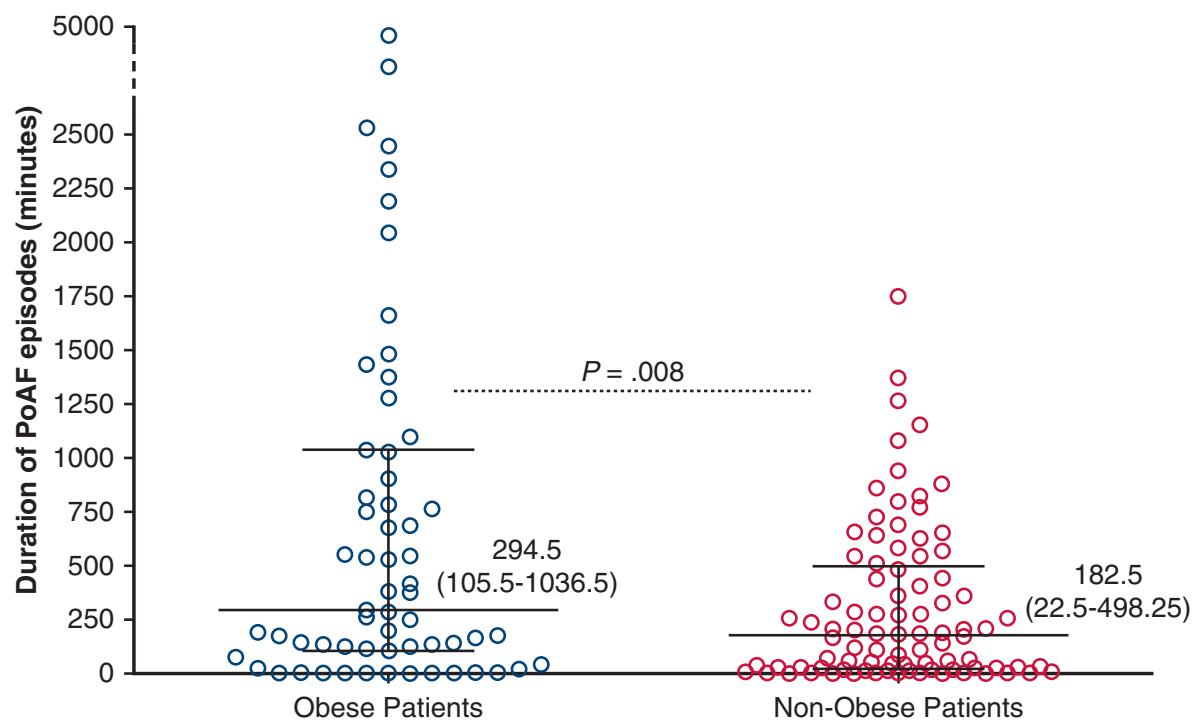

FIGURE 1. Scatter plot demonstrating the relative frequency distribution of the duration of all PoAF episodes. There is a significantly higher duration of early PoAF episodes in obese patients (median, 294.5; IQR, 105.5-1036.5) compared with nonobese patients (median, 182.5; IQR, 22.5-498.25) $(P=.008)$. The thick black lines indicate the median, and the thin lines indicate the IQR (P25/P75). PoAF, Postoperative atrial fibrillation. 


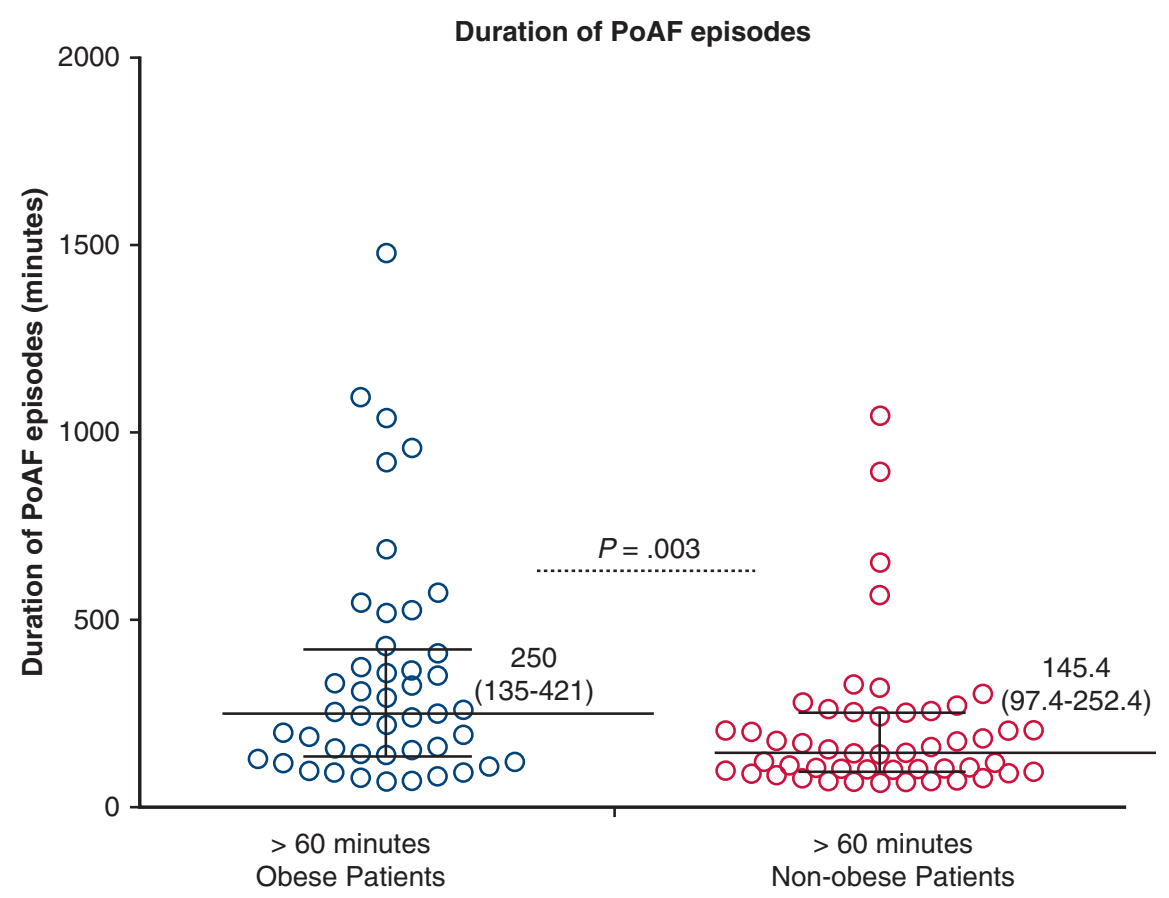

FIGURE 2. Relative frequency distribution of the duration of PoAF episodes lasting longer than 60 minutes. In the obese group, there is a significantly higher median time of prolonged PoAF episodes $(P=.003)$. The thick black lines indicate the median, and the thin lines indicate the IQR $(\mathrm{P} 25 / \mathrm{P} 75)$. PoAF, Postoperative atrial fibrillation.

Variables introduced in the linear regression model of early PoAF burden are shown in Table 3 . The relationship between PoAF burden and possible associated factors is plotted in Figure E1. BMI was the only variable $(\beta 1=0.13, P=.002)$ significantly correlated with early PoAF burden, whereas age, gender, and surgical procedure had no relationship.

Figure E2 shows the PoAF burden and duration of PoAF episodes during the first 5 postoperative days separately. Figure E2 shows that in the obese group the highest PoAF burden was observed on the third postoperative day (median 6.1\%; IQR, 1.3-13.7). In nonobese patients, the highest PoAF burden occurred on the second postoperative day (median 3.5\%; IQR, 0.2-6.1). Figure E2 shows that obese patients spent the longest time in PoAF during the third postoperative day (median 316 minutes; IQR, 93.4-779.3). In nonobese patients, however, the longest time spent in PoAF was on the second postoperative day (median, 194.5 minutes; IQR, 11.8-355.8).

On postoperative day 3 , obese patients had longer PoAF episodes and higher PoAF burden compared with nonobese patients (median, 135.8 minutes; IQR, 18.4-443.9; $P=.035$; median, $2 \%$; IQR, 0.4-6.6; $P=.021$ ).

The relative frequency distribution of prolonged PoAF episodes for each of the first 5 postoperative days is depicted in Table 4. In both obese and nonobese groups, PoAF episodes lasting longer than 60 minutes occurred mainly on the second and third postoperative days. However, there was no significant difference in the incidence of prolonged PoAF episodes on any of the first 5 postoperative days.

\section{Clinical Outcome}

In the obese group, rate-control therapy of PoAF was achieved with antiarrhythmic drugs including $\beta 1$-blockers $(\mathrm{N}=43$ patients, $64 \%$ [43/67]), digoxin ( $\mathrm{N}=10$ patients, $15 \%$ [10/67]), and rhythm control with amiodarone $(\mathrm{N}=15$ patients, $22.3 \%$ [15/67]). In the nonobese group, therapy was aimed at rate control in 79 patients $(\beta 1$ blockers: $\mathrm{N}=63$ patients $[70.7 \%, 63 / 89]$ or digoxin: $\mathrm{N}=16$ patients $[18 \%, 16 / 89])$ or rhythm control in 20 patients $(22.4 \%$ [20/89]). The efficacy of amiodarone in reducing or converting PoAF to sinus rhythm was similar in the obese and nonobese groups $(P=1.000)$. The effect of amiodarone was determined by calculating the difference in median duration of PoAF episodes and PoAF burden between the fifth postoperative day and the day before initiation of treatment.

There was no significant difference in PoAF burden (obese patients: median [-11\%]; IQR [-14.9]-3.1\%; nonobese patients: median [-4\%]; IQR [-9.2]-1.5\%; $P=.059$ ) and duration (obese patients: median -545 ; IQR [-817]-76 minutes; nonobese patients: (median [-319.2]; IQR [-562.2]-103.0 minutes; $P=.298$ ) of PoAF episodes before and after amiodarone administration. Additional electrical cardioversion rhythm was performed in 8 obese patients $(12 \%$ [8/67]) and 6 nonobese patients 

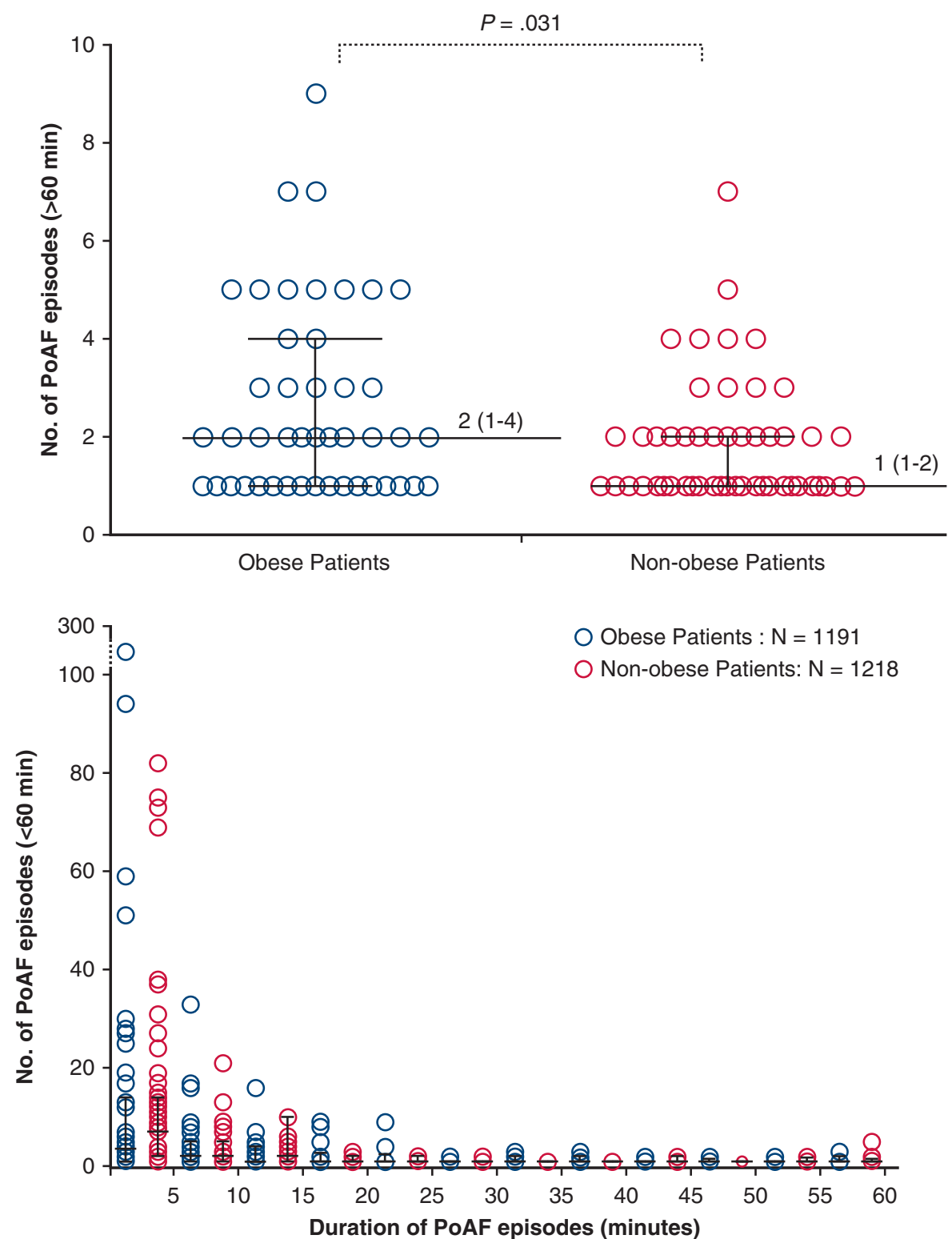

FIGURE 3. Top: the median number of PoAF episodes lasting longer than 60 minutes for obese and nonobese patients separately. There was a significantly higher number of prolonged PoAF episodes in obese patients (1191; median, 2; IQR, 1-4) compared with nonobese patients (1218; median, 1; IQR, 1-2) $(P=.031)$. Bottom: the median number of PoAF episodes categorized individually between 30 seconds and 60 minutes. There was no significant difference in the number of episodes lasting less than 1 hour between obese and nonobese patients. Thick black lines indicate the median, and thin lines indicate the IQR (P25/P75). PoAF, Postoperative atrial fibrillation.

$(6.7 \%[6 / 89])(P=.274)$, resulting in SR in 3 obese patients $(4.4 \%$ [3/67]) and all nonobese patients $(6.7 \%$ [6/89]) $(P=.733)$.

\section{DISCUSSION}

\section{Key Findings}

The present study demonstrated that for patients presenting early PoAF, the burden is significantly higher in obese patients compared with nonobese patients. Overall, long PoAF episodes, lasting between 60 minutes and 24 hours, occurred more frequently in the obese population. These long PoAF episodes occurred mainly on the third postoperative day. There was a significant relationship between BMI and PoAF burden.

\section{Clinical Relevance of De Novo Postoperative Atrial Fibrillation}

De novo early PoAF after cardiac surgery has been associated with increased rates of mortality, morbidity (eg, stroke), and subsequently increased hospitalization time 


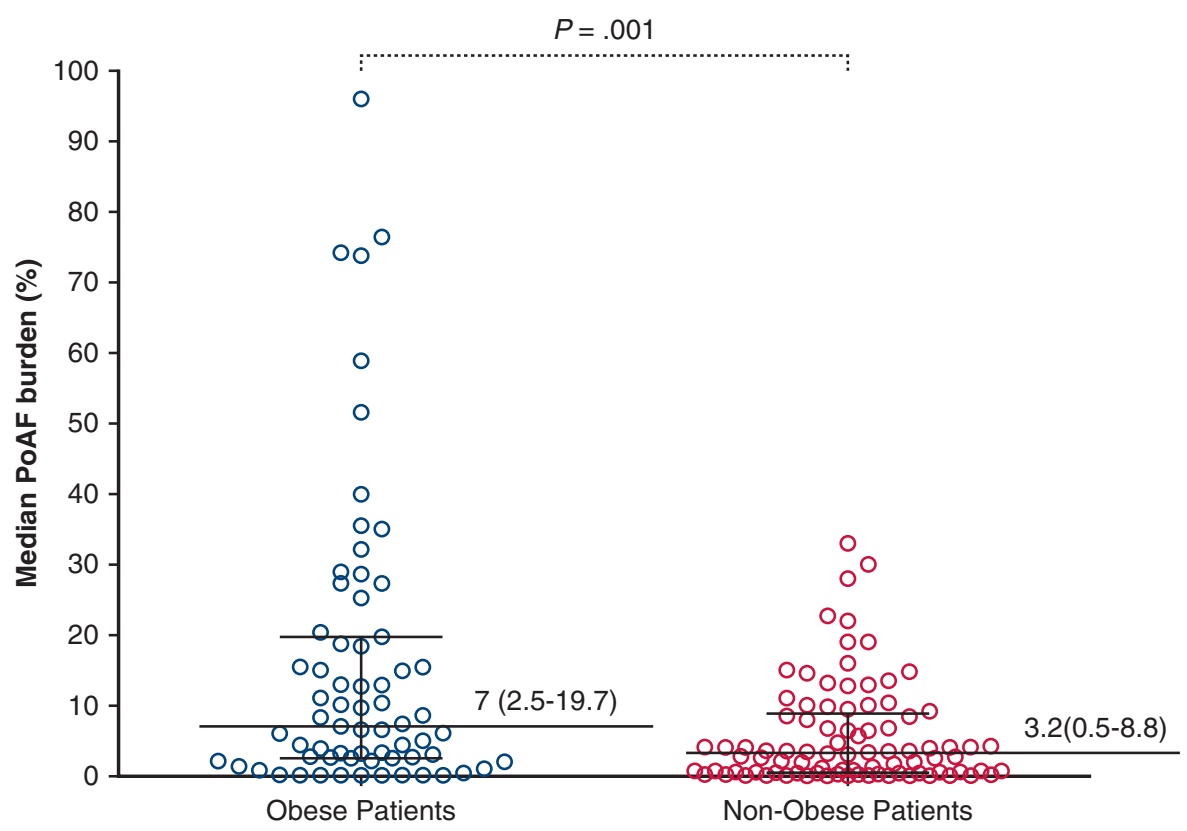

FIGURE 4. Scatter plots demonstrating the relative frequency distribution of the PoAF burden (\%) in obese and nonobese patients separately. There is a significantly higher early PoAF burden in obese patients (median, 7; IQR, 2.5-19.7) compared with nonobese patients (median, 3.2; IQR, 0.5-8.8; $P=.001$ ). Thick black lines indicate the median, and thin lines indicate the IQR (P25/P75). PoAF, Postoperative atrial fibrillation.

and readmissions. Several risk factors contribute to the development of longer duration of PoAF, including older age, male gender, ${ }^{4}$ and obesity. ${ }^{17}$ Intraoperative factors (eg, duration of cardioplegic arrest, postoperative inflammation) may affect atrial electrical properties, thus increasing the likelihood for AF development. ${ }^{4,18}$ Previous studies have demonstrated that de novo onset PoAF predicts development of late PoAF, associated with an increased rate of hospital readmissions, cardiovascular events, and mortality. ${ }^{19}$ The duration of PoAF episodes after cardiac surgery is also associated with decreased long-term survival. ${ }^{20}$ Sigurdsson and colleagues ${ }^{20}$ have demonstrated that survival was significantly worse in patients who experienced PoAF episodes lasting longer than 2 days. In our study, we found that the majority of PoAF episodes lasted less than 5 minutes in both obese $(62.6 \%)$ and nonobese $(68.4 \%)$ patients. Episodes lasting longer than 1 hour were significantly more likely to occur in obese patients

TABLE 3. Multivariate analysis of predictors for early postoperative atrial fibrillation burden

\begin{tabular}{lclc}
\hline \multicolumn{1}{c}{ Variables } & Beta $(\boldsymbol{\beta})$ & $\mathbf{9 5} \%$ CI for $\boldsymbol{\beta}$ & $\boldsymbol{P}$ value \\
\hline BMI & 0.13 & $0.05-0.21$ & .002 \\
Age & 0.02 & -0.02 to 0.06 & .330 \\
Gender & 0.43 & -0.44 to 1.29 & .328 \\
Surgical procedure & 0.09 & -0.24 to 0.42 & .585 \\
\hline
\end{tabular}

Statistically significant values $(P<.05) . C I$, Confidence interval; $B M I$, body mass index.
$(10 \%)$ compared with nonobese patients $(8 \%)$. Thus, the combination between prolonged PoAF episodes and increased associated postoperative morbidity due to obesity $^{21}$ may also severely affect the survival and postoperative quality of life in obese patients.

\section{De Novo Early Postoperative Atrial Fibrillation Burden}

The term "AF burden" is applied to describe the temporal dynamic pattern of AF in terms of presence and duration of AF episodes, as detected by various devices (eg, bedside monitors, Holter monitors, implantable devices). Accurate assessment of AF burden is important because it is related to development of stroke.

Previous studies have demonstrated that BMI, a measure of overall adiposity, is a strong independent associated factor with not only AF but also PoAF. ${ }^{22-24}$ In a meta-analysis of the association between obesity and PoAF in patients without a history of AF, Phan and colleagues ${ }^{24}$ found that obesity was associated with a significant risk of PoAF. However, the de novo early PoAF burden in obese patients has not been assessed. In our study, we found that obese patients had a significantly higher PoAF burden during the first 5 postoperative days when compared with the nonobese patients. Moreover, obese patients experienced more prolonged PoAF episodes, particularly on the third postoperative day. This is an important finding because prior studies have shown that prolonged episodes are associated with a high risk of stroke. ${ }^{25}$ In a pooled analysis performed by 
TABLE 4. Characteristics of postoperative atrial fibrillation burden and duration and number of prolonged ( $>60$ minutes) postoperative atrial fibrillation episodes for each postoperative day separately

\begin{tabular}{|c|c|c|c|c|c|c|c|c|c|c|}
\hline \multirow[b]{2}{*}{ Characteristics of PoAF } & \multicolumn{5}{|c|}{ Obese patients } & \multicolumn{5}{|c|}{ Nonobese patients } \\
\hline & Day 1 & Day 2 & Day 3 & Day 4 & Day 5 & Day 1 & Day 2 & Day 3 & Day 4 & Day 5 \\
\hline \multirow{2}{*}{$\begin{array}{c}\text { PoAF burden }(\%) \\
\text { median (IQR) }\end{array}$} & 0.24 & 3 & 6.1 & 3.83 & 0.42 & 0.15 & 3.5 & 2 & 0.7 & 1.4 \\
\hline & $(0.01-7.37)$ & $(0.36-11.5)$ & $(1.34-13.6)$ & $(0.12-5.72)$ & $(0.05-15.9)$ & $(0.03-0.43)$ & $(0.21-6.14)$ & $(0.35-6.6)$ & $(0.09-2.4)$ & $(0.6-2.8)$ \\
\hline \multirow{2}{*}{$\begin{array}{l}\text { PoAF duration (min) } \\
\text { median (IQR) }\end{array}$} & 12.5 & 175.5 & 316 & 103 & 11 & 6 & 194.7 & 135.7 & 49 & 69 \\
\hline & $(1-430.87)$ & $(24.2-658.2)$ & $(93.3-779.2)$ & $(5.25-273)$ & $(1.5-1375)$ & $(1-28.1)$ & $(11.8-355.8)$ & ) $(18.3-443.8)$ & $(5.62-132.2$ & $(33-150.6)$ \\
\hline \multirow{2}{*}{$\begin{array}{c}\text { Prolonged PoAF } \\
\text { episodes }(\%)\end{array}$} & $6.25 \%$ & $14.05 \%$ & $8.92 \%$ & $12 \%$ & $10 \%$ & $10 \%$ & $15.4 \%$ & $9 \%$ & $6.6 \%$ & $3.8 \%$ \\
\hline & {$[6 / 96]$} & {$[26 / 185]$} & [57/639] & [24/201] & {$[7 / 70]$} & [7/68] & {$[33 / 213]$} & {$[33 / 363]$} & [19/286] & {$[11 / 288]$} \\
\hline
\end{tabular}

$P o A F$, Postoperative atrial fibrillation; $I Q R$, interquartile range.

Boriani and colleagues, ${ }^{26}$ the Cox regression analysis adjusted for Congestive Heart Failure, Hypertension, Age, Diabetes and Stroke/TIA (CHADS2), and anticoagulants at baseline demonstrated that the AF burden was an independent predictor of stroke, and a threshold of 1 hour was associated with the highest hazard ratio for stroke (ie, $2.11 ; 95 \%$ confidence interval, 1.22-3.64; $P=.008$ ).

Our study showed that a 0.13 -unit increase in BMI resulted in an increase in early PoAF burden $(P=.002)$. Although our data showed no significant relationship between PoAF burden and age, gender, or surgical procedure, they could still play an important role in the incidence of early de novo PoAF in a larger population. Other clinical factors such as subcutaneous fat and visceral fat, including epicardial adipose tissue (EAT), could contribute to the increase in PoAF burden in obese patients. EAT has not been assessed in our study, but it is positively correlated with BMI. ${ }^{27}$ EAT also plays an important role in AF pathophysiology by modifying the atrial substrate through the release of inflammatory cytokines and adipokines into adjacent myocardium. ${ }^{28}$ Experimental studies have shown that weight loss can reduce AF burden and obesity-related ionic remodeling.

Age and incidence of DM at baseline were different in our population. Nonobese patients were older than obese patients, and the incidence of DM was significantly lower in the nonobese group (Table 1). However, no relationship was found between early PoAF burden and age or incidence. The remainder of baseline characteristics were similar between the obese and nonobese groups, yet this may depend on the sample size. Hypertension is associated with obesity and is more frequently encountered in patients with PoAF. ${ }^{24,29}$ The majority of our obese patients were hypertensive $(50 \%)$, but the incidence did not differ from the nonobese group.

\section{Therapy of Early Postoperative Atrial Fibrillation}

In our study, we found that amiodarone did not affect the overall number or duration of PoAF episodes. Previous studies have shown that intravenous amiodarone significantly decreases the incidence of PoAF if administered before $^{30}$ or immediately after completion of the surgical procedure. ${ }^{31}$ Further research is needed in assessing whether amiodarone is beneficial in reducing the incidence of the PoAF burden.

\section{Study Limitations}

Our study is limited by the relatively small number of patients included in the study, which may contribute to similarities in baseline characteristics between the obese and nonobese groups. The study may also be limited by exclusive inclusion of patients with early PoAF and the lack of follow-up information after patient discharge from hospital Nevertheless, our data show a clear difference in the characteristics of PoAF between obese and nonobese patients.

\section{CONCLUSIONS}

Obesity predisposes to a larger number of prolonged AF episodes in the early postoperative period after cardiac surgery for CAD or valvular heart disease. The higher AF burden in the early postoperative period occurred particularly on the third day. To determine the impact of the early PoAF burden on long-term clinical outcome, further larger prospective studies are mandatory. Because the effects of obesity on the AF substrate are reversible with weight control, preventive lifestyle before cardiac surgery may play an invaluable role.

\section{Conflict of Interest Statement}

Authors have nothing to disclose with regard to commercial support.

The authors thank Drs Oei, Bekkers, van de Woestijne, van Leeuwen, Taverne, Birim, Mahtab, Bekker, and van Schaagen for their contribution to this work.

\section{References}

1. Go AS, Hylek EM, Phillips KA, Chang Y, Henault LE, Selby JV, et al. Prevalence of diagnosed atrial fibrillation in adults - National implications for rhythm management and stroke prevention: the AnTicoagulation and Risk Factors in Atrial Fibrillation (ATRIA) study. JAMA. 2001;285:2370-5.

2. Ahlsson A, Fengsrud E, Bodin L, Englund A. Postoperative atrial fibrillation in patients undergoing aortocoronary bypass surgery carries an eightfold risk of 
future atrial fibrillation and a doubled cardiovascular mortality. Eur J Cardiothorac Surg. 2010;37:1353-9.

3. Goudis CA, Korantzopoulos P, Ntalas IV, Kallergis EM, Ketikoglou DG. Obesity and atrial fibrillation: a comprehensive review of the pathophysiological mechanisms and links. J Cardiol. 2015;66:361-9.

4. Almassi GH, Schowalter T, Nicolosi AC, Aggarwal A, Moritz TE, Henderson WG, et al. Atrial fibrillation after cardiac surgery: a major morbid event? Ann Surg. 1997; 226:501-13.

5. Lin YK, Chen YJ, Chen SA. Potential atrial arrhythmogenicity of adipocytes: implications for the genesis of atrial fibrillation. Med Hypotheses. 2010;74:1026-9.

6. Savio-Galimberti E. Weight loss reverses ionic remodeling responsible for increased atrial fibrillation inducibility and burden in high-fat diet induced obese mice [Abstract]. presented at: American Heart Association's 2016 Scientific Sessions and Resuscitation Science Symposium; November 12-16, 2016, New Orleans, Louisiana.

7. Savio-Galimberti E, Wasserman D, Darbar D. Weight loss reduces atrial fibrillation inducibility and burden in severe obesity induced by either high-fat diet or genetic hyperphagia in mice [Abstract]. Presented at: American Heart Association's 2015 Scientific Sessions and Resuscitation Science Symposium; November 7-11, 2015, Orlando, Florida.

8. Lauer MS, Eagle KA, Buckley MJ, Desanctis RW. Atrial fibrillation following coronary artery bypass surgery. Prog Cardiovasc Dis. 1989;31:367-78.

9. Hravnak M, Hoffman LA, Saul MI, Zullo TG, Whitman GR, Griffith BP. Predictors and impact of atrial fibrillation after isolated coronary artery bypass grafting Crit Care Med. 2002;30:330-7.

10. Zaman AG, Archbold RA, Helft G, Paul EA, Curzen NP, Mills PG. Atrial fibrillation after coronary artery bypass surgery: a model for preoperative risk stratification. Circulation. 2000;101:1403-8.

11. Mathew JP, Parks R, Savino JS, Friedman A, Koch C, Mangano DT, et al. Atrial fibrillation following coronary artery bypass graft surgery-predictors, outcomes, and resource utilization. JAMA. 1996;276:300-6.

12. Amar D, Shi W, Hogue CW, Zhang H, Passman RS, Thomas B, et al. Clinical prediction rule for atrial fibrillation after coronary artery bypass grafting. J Am Coll Cardiol. 2004:44:1248-53.

13. Yaksh A, Kik C, Knops P, van Ettinger MJ, Bogers AJ, de Groot NM. Early, de novo atrial fibrillation after coronary artery bypass grafting: facts and features. Am Heart J. 2017;184:62-70.

14. Zacharias A, Schwann TA, Riordan CJ, Durham SJ, Shah AS, Habib RH. Obesity and risk of new-onset atrial fibrillation after cardiac surgery. Circulation. 2005; 112:3247-55.

15. Kuduvalli M, Grayson AD, Oo AY, Fabri BM, Rashid A. Risk of morbidity and in-hospital mortality in obese patients undergoing coronary artery bypass surgery. Eur J Cardiothorac Surg. 2002:22:787-93.

16. Gao M, Sun J, Young N, Boyd D, Atkins Z, Li Z, et al. Impact of body mass index on outcomes in cardiac surgery. J Cardiothorac Vasc Anesth. 2016;30:1308-16.

17. Sun X, Boyce SW, Hill PC, Bafi AS, Xue Z, Lindsay J, et al. Association of body mass index with new-onset atrial fibrillation after coronary artery bypass grafting operations. Ann Thorac Surg. 2011;91:1852-8.
18. Mathew JP, Fontes ML, Tudor IC, Ramsey J, Duke P, Mazer CD, et al. A multi center risk index for atrial fibrillation after cardiac surgery. JAMA. 2004;291 $1720-9$

19. Lee SH, Kang DR, Uhm JS, Shim J, Sung J-H, Kim J-Y, et al. New-onset atrial fibrillation predicts long-term newly developed atrial fibrillation after coronary artery bypass graft. Am Heart J. 2014;167:593-600 e591.

20. Sigurdsson MI, Longford NT, Heydarpour M, Saddic L, Chang T-W, Fox AA et al. Duration of postoperative atrial fibrillation after cardiac surgery is associated with worsened long-term survival. Ann Thorac Surg. 2016;102:2018-26.

21. Jongnarangsin K, Chugh A, Good E, Mukerji S, Dey S, Crawford T, et al. Body mass index, obstructive sleep apnea, and outcomes of catheter ablation of atria fibrillation. J Cardiovasc Electrophysiol. 2008;19:668-72.

22. Esato M, Shimizu A, Chun YH, Tatsuno H, Yamagata T, Matsuzaki M. Electrophysiologic effects of a class I antiarrhythmic agent, cibenzoline, on the refractoriness and conduction of the human atrium in vivo. J Cardiovasc Pharmacol. 1996;28:321-7

23. Wang TJ, Parise H, Levy D, D'Agostino RB, Wolf PA, Vasan RS, et al. Obesity and the risk of new-onset atrial fibrillation. JAMA. 2004;292:2471-7.

24. Phan K, Khuong JN, Xu J, Kanagaratnam A, Yan TD. Obesity and postoperative atrial fibrillation in patients undergoing cardiac surgery: systematic review and meta-analysis. Int J Cardiol. 2016;217:49-57.

25. Boriani G, Diemberger I, Ziacchi M, Valzania C, Gardini B, Cimaglia P, et al. AF burden is important - fact or fiction? Int J Clin Pract. 2014;68:444-52.

26. Boriani G, Glotzer TV, Santini M, West TM, De Melis M, Sepsi M, et al. Devicedetected atrial fibrillation and risk for stroke: an analysis of $>10,000$ patient from the SOS AF project (Stroke preventiOn Strategies based on Atrial Fibrillation information from implanted devices). Eur Heart J. 2014;35:508-16.

27. Rabkin SW. The relationship between epicardial fat and indices of obesity and the metabolic syndrome: a systematic review and meta-analysis. Metab Syndr Relat Disord. 2014;12:31-42.

28. Zghaib T, Ipek EG, Zahid S, Balouch MA, Misra S, Ashicaga H, et al. Association of left atrial epicardial adipose tissue with electrogram bipolar voltage and fractionation: electrophysiologic substrates for atrial fibrillation. Heart Rhythm. 2016;13:2333-9.

29. Stamou SC, Nussbaum M, Stiegel RM, Reames MK, Skipper ER, Robicsek F, et al. Effect of body mass index on outcomes after cardiac surgery: is there an obesity paradox? Ann Thorac Surg. 2011;91:42-7.

30. Guarnieri T, Nolan S, Gottlieb SO, Dudek A, Lowry DR. Intravenous amiodarone for the prevention of atrial fibrillation after open heart surgery: the Amiodarone Reduction in Coronary Heart (ARCH) trial. J Am Coll Cardiol. 1999;34:343-7.

31. Hohnloser SH, Meinertz T, Dammbacher T, Steiert K, Jahnchen E, Zehender M, et al. Electrocardiographic and antiarrhythmic effects of intravenous amiodarone: results of a prospective, placebo-controlled study. Am Heart J. 1991; 121(1 Pt 1):89-95

Key Words: cardiac surgery, de novo early postoperative atrial fibrillation, obesity 

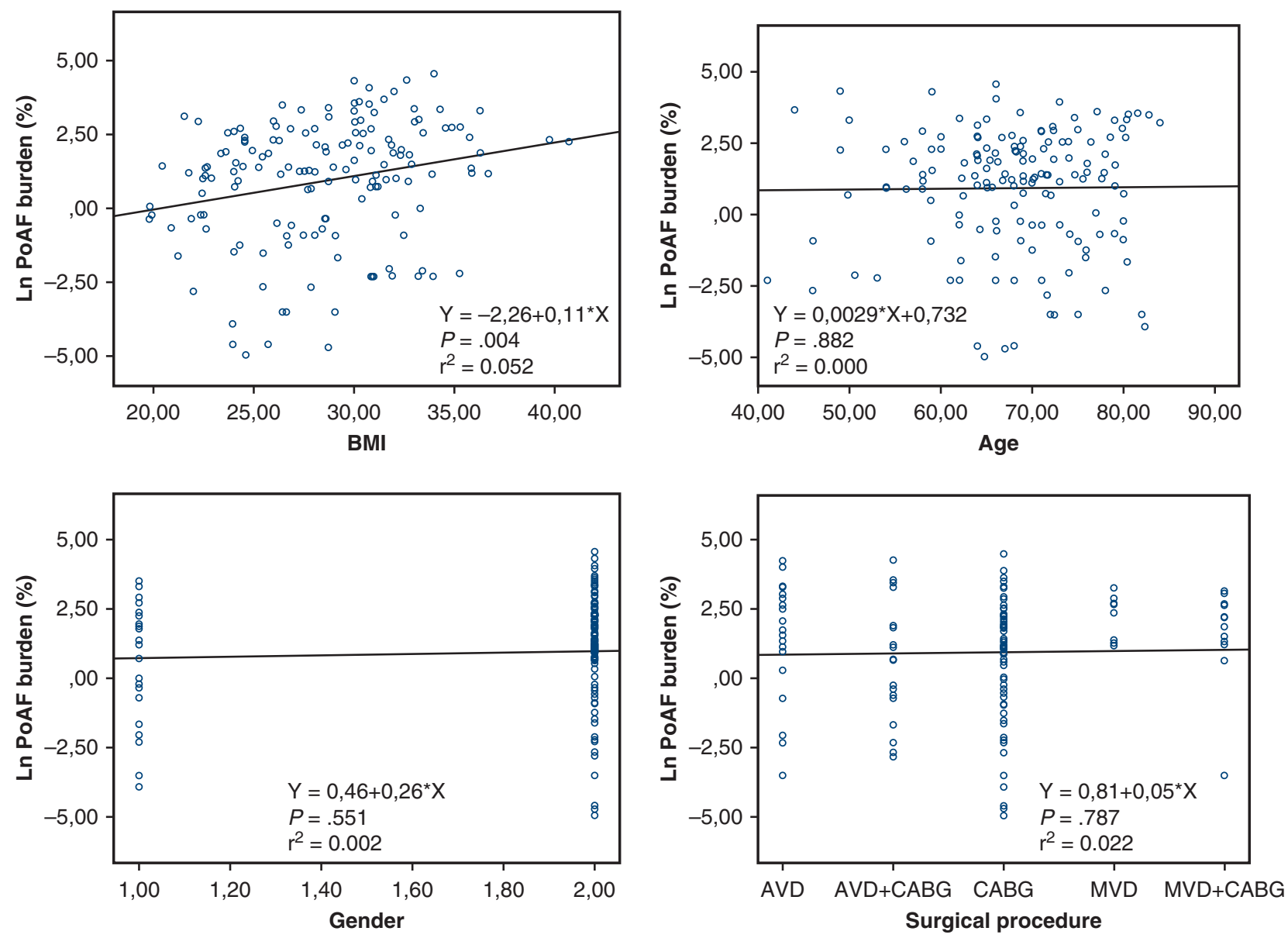

FIGURE E1. Scatterplots of the linear correlations and their intercepts between logarithm of PoAF and independent variables. Top left: the relationship between BMI and PoAF burden. There is a small $\left(\mathrm{r}^{2}=0.052\right)$ but significant linear association between BMI and PoAF $(P=.004)$. Top right: the relationship between age and PoAF burden. There is no significant association between the 2 variables $\left(\mathrm{r}^{2}=0.000, P=.882\right)$. Lower left and right: the association between PoAF burden and gender and surgical procedure, respectively. No significant relationship was found between gender $\left(\mathrm{r}^{2}=0.002 ; P=.551\right)$ or surgical procedure $\left(\mathrm{r}^{2}=0.022 ; P=.787\right)$ and PoAF burden. $L n P o A F$, Logarithm of postoperative atrial fibrillation; $A V D$, atrial valve disease; $C A B G$, coronary artery bypass grafting; $M V D$, mitral valve disease; $r^{2}$, coefficient of determination. 

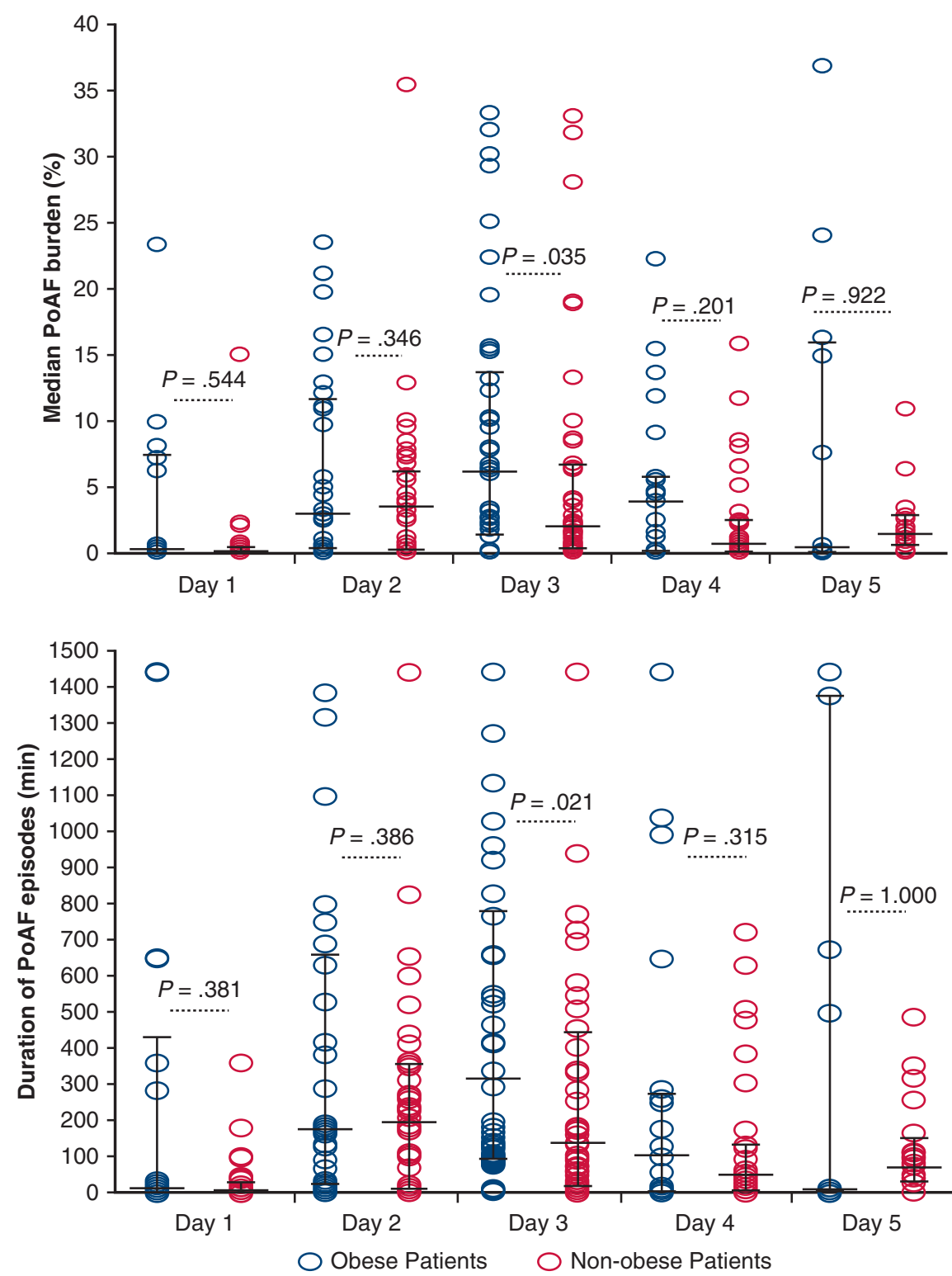

FIGURE E2. Daily analysis of PoAF episodes lasting more than 60 minutes. Top: The plots represent the relative frequency distribution of PoAF burden for each of the 5 postoperative days individually. On postoperative day 3, there is an increase in the PoAF burden in the obese group (median, $6.1 \%$; IQR, 1.3 13.6) compared with the nonobese group (median, 2; IQR, 0.4-6.6) $(P=.031)$. Bottom: The relative frequency distribution of the median time spent in $\mathrm{AF}$ on each of the 5 postoperative days individually. Obese patients (median, 316 minutes; IQR, 93.3-779.2) spent a longer time in AF compared with nonobese patients (median, 135.7 minutes; IQR, 18.3-443.8) $(P=.021)$ during postoperative day 3. PoAF, Postoperative atrial fibrillation. 